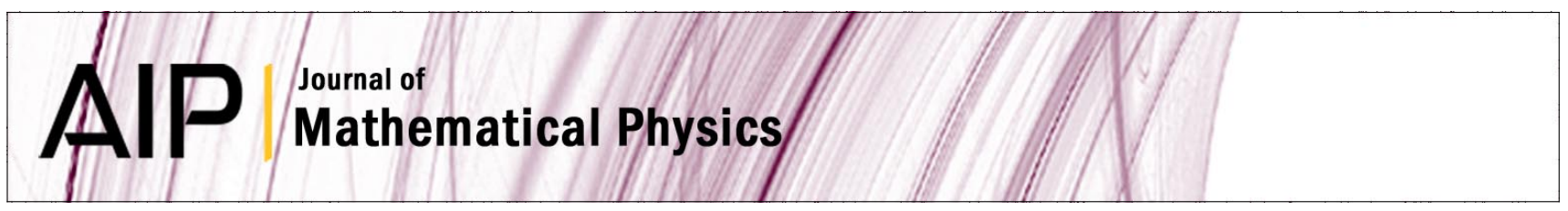

\title{
Infinitely many periodic orbits for the rhomboidal five-body problem
}

Montserrat Corbera and Jaume Llibre

Citation: J. Math. Phys. 47, 122701 (2006); doi: 10.1063/1.2378617

View online: http://dx.doi.org/10.1063/1.2378617

View Table of Contents: http://jmp.aip.org/resource/1/JMAPAQ/v47/i12

Published by the American Institute of Physics.

\section{Related Articles}

Dziobek configurations of the restricted $(\mathrm{N}+1)$-body problem with equal masses J. Math. Phys. 53, 072902 (2012)

Laser heating of finite two-dimensional dust clusters: B. Simulations

Phys. Plasmas 19, 023701 (2012)

Super central configurations of the three-body problem under the inverse integer power law J. Math. Phys. 52, 092901 (2011)

Thomas-forbidden particle capture

J. Math. Phys. 52, 062901 (2011)

Scattering of scalar waves by many small particles

AlP Advances 1, 022135 (2011)

\section{Additional information on J. Math. Phys.}

Journal Homepage: http://jmp.aip.org/

Journal Information: http://jmp.aip.org/about/about_the_journal

Top downloads: http://jmp.aip.org/features/most_downloaded

Information for Authors: http://jmp.aip.org/authors

\section{ADVERTISEMENT}

The most comprehensive support for physics in any mathematical software package World-leading tools for performing calculations in theoretical physics

www.maplesoft.com/physics
Your work in Maple matches how you would write the problems and solutions by hand

State-of-the-art environment for algebraic computations in physics

The only system with the ability to handle a wide range of physics computations as well as pencil-and-paper style input and textbook-quality display of results

- Access to Maple's full mathematical power, programming language, visualization routines, and document creation tools 


\title{
Infinitely many periodic orbits for the rhomboidal five-body problem
}

\author{
Montserrat Corbera ${ }^{a}$ \\ Department of Digital and Information Technologies, University of Vic, Laura 13, \\ 08500 Vic, Barcelona, Spain \\ Jaume Llibre ${ }^{\text {b) }}$ \\ Department of Mathematics, Autonomous, University of Barcelona, 08193 Bellaterra, \\ Barcelona, Spain
}

(Received 20 July 2006; accepted 5 October 2006; published online 8 December 2006)

\begin{abstract}
We prove the existence of infinitely many symmetric periodic orbits for a regularized rhomboidal five-body problem with four small masses placed at the vertices of a rhombus centered in the fifth mass. The main tool for proving the existence of such periodic orbits is the analytic continuation method of Poincaré together with the symmetries of the problem. (C) 2006 American Institute of Physics.
\end{abstract}

[DOI: $10.1063 / 1.2378617]$

\section{INTRODUCTION}

In this paper we consider a particular case of the planar five-body problem defined as follows. We consider a mass $m_{0}=1$ at the origin of coordinates with zero initial velocity, two small masses $m_{1}=m_{2}=\mu \nu_{1}$ with initial positions and velocities on the $x$ axis symmetric with respect to the origin, and two small masses $m_{3}=m_{4}=\mu \nu_{2}$ with initial positions and velocities on the $y$ axis also symmetric with respect to the origin (see Fig. 1). Our five-body problem consists of describing the motion of the five masses under their mutual Newtonian gravitational attraction. Due to the symmetry of the initial conditions and velocities, the four small bodies form a rhombus with center at $m_{0}$ at any time and the mass $m_{0}$ remains at rest at the origin. The description of the motion of this five-body problem is called the rhomboidal five-body problem.

Although this is a five-body problem it can be formulated as a Hamiltonian system of two degrees of freedom, one is the distance $x \geqslant 0$ of $m_{1}$ to the origin and the other is the distance $y$ $\geqslant 0$ of $m_{3}$ to the origin (the distances of $m_{2}$ and $m_{4}$ to the origin are obtained by symmetry). The system has three singularities, the triple collision between $m_{0}, m_{1}$, and $m_{2}$, the triple collision between $m_{0}, m_{3}$, and $m_{4}$, and the total collision of the five bodies. Due to the symmetries doing a double Levi-Civita transformation we regularize both triple collisions.

When $\mu=0$ the problem is reduced to two collision two-body problems, the collision twobody problem with $m_{0}$ and $m_{1}$ and the collision two-body problem with $m_{0}$ and $m_{3}$. Note that if we take into account the five bodies, then really for $\mu=0$ we have instead of the binary collisions $m_{0}$ with $m_{1}$, and $m_{0}$ with $m_{3}$, the triple collisions $m_{0}, m_{1}$, and $m_{2}$, and $m_{0}, m_{3}$, and $m_{4}$. Since the solutions of the collision two-body problem are known we can compute the periodic solutions of the regularized system for $\mu=0$ in a fixed energy level $h<0$. The objective of this paper is to prove that the symmetric periodic orbits of the regularized rhomboidal five-body problem for $\mu$ $=0$ can be continued to symmetric periodic orbits of the regularized rhomboidal five-body problem for $\mu>0$ sufficiently small. The main tool for proving this result is the classical analytic continuation method of Poincaré.

\footnotetext{
${ }^{a)}$ Electronic mail: montserrat.corbera@uvic.cat

${ }^{b)}$ Electronic mail: jllibre@mat.uab.cat
} 


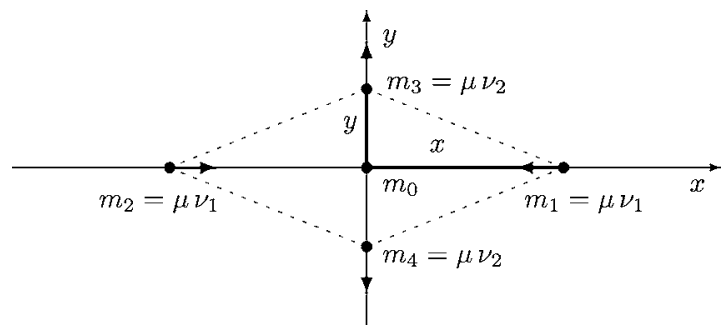

FIG. 1. The rhomboidal five-body problem.

The paper is structured as follows. In Sec. II we give the equations of motion of the rhomboidal five-body problem and we apply a double Levi-Civita transformation to regularize the triple collision between $m_{0}, m_{1}$, and $m_{2}$ and the triple collision between $m_{0}, m_{3}$, and $m_{4}$. Notice that the total collision of the five bodies is not regularized. In Sec. III we analyze the discrete symmetries of the regularized problem. In particular we see that there are three different symmetries that provide symmetric periodic solutions of the problem. We characterize these symmetric periodic solutions, and the double symmetric periodic solutions which are the main objective of this work. In Sec. IV we compute the periodic solutions of the regularized rhomboidal five-body problem for $\mu=0$ and in particular we analyze the double symmetric periodic solutions. Finally in Sec. V we apply the analytic continuation method of Poincaré to continue the double symmetric periodic orbits of the regularized rhomboidal five-body problem for $\mu=0$ to double-symmetric periodic orbits of the regularized rhomboidal five-body problem for $\mu>0$ sufficiently small.

\section{EQUATIONS OF MOTION FOR THE RHOMBOIDAL FIVE-BODY PROBLEM}

We consider five point particles with masses $m_{0}=1, m_{1}=m_{2}=\mu \nu_{1}, m_{3}=m_{4}=\mu \nu_{2}$, positions $\mathbf{q}_{0}=(0,0), \mathbf{q}_{1}=(x, 0), \mathbf{q}_{2}=(-x, 0), \mathbf{q}_{3}=(0, y)$, and $\mathbf{q}_{4}=(0,-y)$, respectively, and velocities $\mathbf{v}_{0}$ $=(0,0), \mathbf{v}_{1}=\left(v_{x}, 0\right), \mathbf{v}_{2}=\left(-v_{x}, 0\right), \mathbf{v}_{3}=\left(0, v_{y}\right)$, and $\mathbf{v}_{4}=\left(0,-v_{y}\right)$, respectively (see Fig. 1). Our fivebody problem consist of describing the motion of these particles under their mutual Newtonian gravitational attraction. We note that due to the symmetry of the problem the mass $m_{0}$ is at rest at the origin and the motion of the masses $m_{1}$ and $m_{2}$ (respectively, $m_{3}$ and $m_{4}$ ) is confined to the $x$ axis (respectively, $y$ axis). Since the configuration of the four bodies in motion is always a rhombus with center at $m_{0}$, we call the five-body problem the rhomboidal five-body problem.

Without loss of generality we can assume that the gravitational constant is $G=1$. Then the kinetic energy of the rhomboidal five-body problem is

$$
T=\mu \nu_{1} \dot{x}^{2}+\mu \nu_{2} \dot{y}^{2}
$$

where the dot denotes derivative with respect to the time $t$ and the potential energy is

$$
U=-\frac{\mu \nu_{1}\left(4+\mu \nu_{1}\right)}{2 x}-\frac{\mu \nu_{2}\left(4+\mu \nu_{2}\right)}{2 y}-\frac{4 \mu^{2} \nu_{1} \nu_{2}}{\sqrt{x^{2}+y^{2}}}
$$

The Lagrangian of the problem is given by $L=T-U$. By the Legendre transformation (see, for instance, Refs. 1-3) the Hamiltonian of the problem is

$$
H=\frac{p_{x}^{2}}{4 \mu \nu_{1}}+\frac{p_{y}^{2}}{4 \mu \nu_{2}}-\frac{\mu \nu_{1}\left(4+\mu \nu_{1}\right)}{2 x}-\frac{\mu \nu_{2}\left(4+\mu \nu_{2}\right)}{2 y}-\frac{4 \mu^{2} \nu_{1} \nu_{2}}{\sqrt{x^{2}+y^{2}}},
$$

where $p_{x}$ and $p_{y}$ are the conjugate momenta. The equations of motion associated to the Hamiltonian $H$ are

$$
\dot{x}=\frac{p_{x}}{2 \mu \nu_{1}}, \quad \dot{p}_{x}=-\frac{\mu \nu_{1}\left(4+\mu \nu_{1}\right)}{2 x^{2}}-\frac{4 \mu^{2} \nu_{1} \nu_{2} x}{\left(x^{2}+y^{2}\right)^{3 / 2}},
$$




$$
\dot{y}=\frac{p_{y}}{2 \mu \nu_{2}}, \quad \dot{p}_{y}=-\frac{\mu \nu_{2}\left(4+\mu \nu_{2}\right)}{2 y^{2}}-\frac{4 \mu^{2} \nu_{1} \nu_{2} y}{\left(x^{2}+y^{2}\right)^{3 / 2}} .
$$

Doing the rescaling of the variables $x=\mu^{2} X, y=\mu^{2} Y$, and $t=\mu^{3} T$, and denoting the new variables $(X, Y, T)$ again by $(x, y, t)$ system (1) becomes

$$
\begin{aligned}
& \dot{x}=\frac{p_{x}}{2 \nu_{1}}, \quad \dot{p}_{x}=-\frac{\nu_{1}\left(4+\mu \nu_{1}\right)}{2 x^{2}}-\frac{4 \mu \nu_{1} \nu_{2} x}{\left(x^{2}+y^{2}\right)^{3 / 2}}, \\
& \dot{y}=\frac{p_{y}}{2 \nu_{2}}, \quad \dot{p}_{y}=-\frac{\nu_{2}\left(4+\mu \nu_{2}\right)}{2 y^{2}}-\frac{4 \mu \nu_{1} \nu_{2} y}{\left(x^{2}+y^{2}\right)^{3 / 2}} .
\end{aligned}
$$

This system is also Hamiltonian with Hamiltonian

$$
H=\frac{p_{x}^{2}}{4 \nu_{1}}+\frac{p_{y}^{2}}{4 \nu_{2}}-\frac{\nu_{1}\left(4+\mu \nu_{1}\right)}{2 x}-\frac{\nu_{2}\left(4+\mu \nu_{2}\right)}{2 y}-\frac{4 \mu \nu_{1} \nu_{2}}{\sqrt{x^{2}+y^{2}}}
$$

We note that system (2) has three singularities: $x=0$, that corresponds to triple collision between $m_{0}, m_{1}$, and $m_{2}, y=0$ that corresponds to triple collision between $m_{0}, m_{3}$, and $m_{4}$, and finally $x^{2}+y^{2}=0$ that corresponds to the total collision of the five bodies. We regularize both triple collisions applying a double Levi-Civita transformation (see Refs. 4-6)

$$
x=\xi_{1}^{2}, \quad y=\xi_{2}^{2}, \quad p_{x}=\frac{\eta_{1}}{2 \xi_{1}}, \quad p_{y}=\frac{\eta_{2}}{2 \xi_{2}}, \quad d t=4 \xi_{1}^{2} \xi_{2}^{2} d s .
$$

The regularized system of the rhomboidal five-body problem (2) on the level energy $H=h$ for some constant $h$ is the Hamiltonian system

$$
\begin{gathered}
\frac{d \xi_{1}}{d s}=\frac{\eta_{1} \dot{\xi}_{2}^{2}}{2 \nu_{1}}, \\
\frac{d \xi_{2}}{d s}=\frac{\eta_{2} \xi_{1}^{2}}{2 \nu_{2}}, \\
\frac{d \eta_{1}}{d s}=-\frac{\xi_{1} \eta_{2}^{2}}{2 \nu_{2}}+8 h \xi_{1} \xi_{2}^{2}+4 \nu_{2}\left(4+\mu \nu_{2}\right) \xi_{1}+\frac{32 \mu \nu_{1} \nu_{2} \xi_{1} \xi_{2}^{6}}{\left(\xi_{1}^{4}+\xi_{2}^{4}\right)^{3 / 2}}, \\
\frac{d \eta_{2}}{d s}=-\frac{\xi_{2} \eta_{1}^{2}}{2 \nu_{1}}+8 h \xi_{1}^{2} \xi_{2}+4 \nu_{1}\left(4+\mu \nu_{1}\right) \xi_{2}+\frac{32 \mu \nu_{1} \nu_{2} \xi_{1}^{6} \xi_{2}}{\left(\xi_{1}^{4}+\xi_{2}^{4}\right)^{3 / 2}}
\end{gathered}
$$

with Hamiltonian

$$
K=\frac{\eta_{1}^{2} \xi_{2}^{2}}{4 \nu_{1}}+\frac{\eta_{2}^{2} \xi_{1}^{2}}{4 \nu_{2}}-2 \nu_{2}\left(4+\mu \nu_{2}\right) \xi_{1}^{2}-2 \nu_{1}\left(4+\mu \nu_{1}\right) \xi_{2}^{2}-4 h \xi_{1}^{2} \xi_{2}^{2}-\frac{16 \mu \nu_{1} \nu_{2} \xi_{1}^{2} \xi_{2}^{2}}{\sqrt{\xi_{1}^{4}+\xi_{2}^{4}}}
$$

and satisfying the energy relation $K=0$; i.e., $H=h$.

We note that system (3) is analytic with respect to its variables except when $\xi_{1}^{4}+\xi_{2}^{4}=0$ which corresponds to the total collision.

The regularization of the triple collisions allows us to look for periodic orbits of the rhomboidal five-body problem containing triple collisions between $m_{0}, m_{1}$, and $m_{2}$ and between $m_{0}$ and $m_{3}$ and $m_{4}$. Our aim is to find periodic orbits of the rhomboidal five-body problem (3) for $\mu>0$ sufficiently small, satisfying the energy relation $K=0$. In fact, we look only for symmetric periodic orbits which are easier to study than the general periodic orbits. 


\section{SYMMETRIES}

It is easy to check that system (3) is invariant under the discrete symmetries

$$
\begin{gathered}
I d: \quad\left(\xi_{1}, \xi_{2}, \eta_{1}, \eta_{2}, s\right) \rightarrow\left(\xi_{1}, \xi_{2}, \eta_{1}, \eta_{2}, s\right), \\
S_{1}: \quad\left(\xi_{1}, \xi_{2}, \eta_{1}, \eta_{2}, s\right) \rightarrow\left(-\xi_{1}, \xi_{2}, \eta_{1},-\eta_{2},-s\right), \\
S_{2}: \quad\left(\xi_{1}, \xi_{2}, \eta_{1}, \eta_{2}, s\right) \rightarrow\left(\xi_{1},-\xi_{2},-\eta_{1}, \eta_{2},-s\right), \\
S_{3}: \quad\left(\xi_{1}, \xi_{2}, \eta_{1}, \eta_{2}, s\right) \rightarrow\left(\xi_{1}, \xi_{2},-\eta_{1},-\eta_{2},-s\right), \\
S_{4}: \quad\left(\xi_{1}, \xi_{2}, \eta_{1}, \eta_{2}, s\right) \rightarrow\left(-\xi_{1},-\xi_{2},-\eta_{1},-\eta_{2}, s\right), \\
S_{5}: \quad\left(\xi_{1}, \xi_{2}, \eta_{1}, \eta_{2}, s\right) \rightarrow\left(-\xi_{1}, \xi_{2},-\eta_{1}, \eta_{2}, s\right), \\
S_{6}: \quad\left(\xi_{1}, \xi_{2}, \eta_{1}, \eta_{2}, s\right) \rightarrow\left(\xi_{1},-\xi_{2}, \eta_{1},-\eta_{2}, s\right), \\
S_{7}: \quad\left(\xi_{1}, \xi_{2}, \eta_{1}, \eta_{2}, s\right) \rightarrow\left(-\xi_{1},-\xi_{2}, \eta_{1}, \eta_{2},-s\right) .
\end{gathered}
$$

The invariance under these symmetries means that if $\varphi(s)=\left(\xi_{1}(s), \xi_{2}(s), \eta_{1}(s), \eta_{2}(s)\right)$ is a solution of system (3), then also $S_{i}(\varphi(s))$ is a solution for $i=1, \ldots, 7$. An orbit $\varphi(s)$ is called $S_{i}$-symmetric if $S_{i}(\varphi(s))=\varphi(s)$.

We note that $\left\{I d, S_{1}, \ldots, S_{7}\right\}$ with the usual composition forms an abelian group isomorphic to $Z_{2} \times Z_{2} \times Z_{2}$. This discrete group of symmetries appeared in many Hamiltonian systems as, for instance, the anisotropic Kepler problem, ${ }^{7}$ the Manev anisotropic problem, ${ }^{8}$ or the collinear threebody problem. ${ }^{9}$

Using the uniqueness theorem of a solution of an ordinary differential system, it follows easily that $\varphi(s)$ is a $S_{1}$-symmetric solution if and only if $\varphi(s)$ intersects the plane $\xi_{1}=\eta_{2}=0$ at least in one point. Now, it is clear that a periodic solution is $S_{1}$-symmetric if and only if it has exactly two intersection points with the plane $\xi_{1}=\eta_{2}=0$. So, clearly the half-period of such a $S_{1}$-symmetric periodic orbit is the time which the orbit needs for travel from one of the intersection points to the other. Using similar arguments for the other symmetries, we obtain the following proposition.

Proposition 1: Let $\varphi(s)=\left(\xi_{1}(s), \xi_{2}(s), \eta_{1}(s), \eta_{2}(s)\right)$ be a solution of system (3).

(a) If $\xi_{1}(s)$ and $\eta_{2}(s)$ are zero at $s=s_{0}$ and at $s=s_{0}+S / 2$ but they are not simultaneously zero at any value of $s \in\left(s_{0}, s_{0}+S / 2\right)$, then $\varphi(s)$ is a $S_{1}$-symmetric periodic solution of period $S$. (b) If $\xi_{2}(s)$ and $\eta_{1}(s)$ are zero at $s=s_{0}$ and at $s=s_{0}+S / 2$ but they are not simultaneously zero at any value of $s \in\left(s_{0}, s_{0}+S / 2\right)$, then $\varphi(s)$ is a $S_{2}$-symmetric periodic solution of period $S$. (c) If $\eta_{1}(s)$ and $\eta_{2}(s)$ are zero at $s=s_{0}$ and at $s=s_{0}+S / 2$ but they are not simultaneously zero at any value of $s \in\left(s_{0}, s_{0}+S / 2\right)$, then $\varphi(s)$ is a $S_{3}$-symmetric periodic solution of period $S$.

Since in system (3) the total collision is not regularized, in our study we must avoid the orbits of the rhomboidal five-body problem which start or end in total collision. In the variables that we are working the total collision takes place when $\xi_{1}=\xi_{2}=0$. Therefore, the symmetries $S_{4}$ and $S_{7}$ are not considered because their symmetric orbits present total collision. Due to the fact that $S_{5}=S_{1}$ - $S_{3}$ and $S_{6}=S_{2} \circ S_{3}$, studying the symmetric periodic orbits with respect to $S_{1}, S_{2}$, and $S_{3}$ we shall get also the symmetric periodic orbits with respect to $S_{5}$ and $S_{6}$.

There could be periodic solutions of system (3) that are simultaneously $S_{1}$ - and $S_{2}$-symmetric periodic solutions. These periodic solutions will be called $S_{12}$-symmetric periodic solutions. In a similar way we can define the $S_{13}$-symmetric periodic solutions and the $S_{23}$-symmetric periodic solutions. These kinds of symmetric periodic solutions are characterized in the following result. 
Proposition 2: Let $\varphi(s)=\left(\xi_{1}(s), \xi_{2}(s), \eta_{1}(s), \eta_{2}(s)\right)$ be a solution of rhomboidal five-body problem (3).

(a) The solution $\varphi(s)$ is a $S_{12}$-symmetric periodic solution of period $S$ if and only if either $\xi_{1}\left(s_{0}\right)=\eta_{2}\left(s_{0}\right)=0$, and $\xi_{2}\left(s_{0}+S / 4\right)=\eta_{1}\left(s_{0}+S / 4\right)=0$, and there is no $s \in\left(s_{0}, s_{0}+S / 4\right)$ such that $\xi_{2}(s)=\eta_{1}(s)=0$; or $\xi_{2}\left(s_{0}\right)=\eta_{1}\left(s_{0}\right)=0$, and $\xi_{1}\left(s_{0}+S / 4\right)=\eta_{2}\left(s_{0}+S / 4\right)=0$, and there is no $s \in\left(s_{0}, s_{0}+S / 4\right)$ such that $\xi_{1}(s)=\eta_{2}(s)=0$.

(b) The solution $\varphi(s)$ is a $S_{13}$-symmetric periodic solution of period $S$ if and only if either $\xi_{1}\left(s_{0}\right)=\eta_{2}\left(s_{0}\right)=0$, and $\eta_{1}\left(s_{0}+S / 4\right)=\eta_{2}\left(s_{0}+S / 4\right)=0$, and there is no $s \in\left(s_{0}, s_{0}+S / 4\right)$ such that $\eta_{1}(s)=\eta_{2}(s)=0 ;$ or $\eta_{1}\left(s_{0}\right)=\eta_{2}\left(s_{0}\right)=0$, and $\xi_{1}\left(s_{0}+S / 4\right)=\eta_{2}\left(s_{0}+S / 4\right)=0$, and there is no $s \in\left(s_{0}, s_{0}+S / 4\right)$ such that $\xi_{1}(s)=\eta_{2}(s)=0$.

(c) The solution $\varphi(s)$ is a $S_{23}$-symmetric periodic solution of period $S$ if and only if either $\xi_{2}\left(s_{0}\right)=\eta_{1}\left(s_{0}\right)=0$, and $\eta_{1}\left(s_{0}+S / 4\right)=\eta_{2}\left(s_{0}+S / 4\right)=0$, and there is no $s \in\left(s_{0}, s_{0}+S / 4\right)$ such that $\eta_{1}(s)=\eta_{2}(s)=0$; or $\eta_{1}\left(s_{0}\right)=\eta_{2}\left(s_{0}\right)=0$, and $\xi_{2}\left(s_{0}+S / 4\right)=\eta_{1}\left(s_{0}+S / 4\right)=0$, and there is no $s \in\left(s_{0}, s_{0}+S / 4\right)$ such that $\xi_{2}(s)=\eta_{1}(s)=0$.

The next result shows that there are no symmetric periodic orbits with respect more than two symmetries.

Proposition 3: There are no periodic solutions of the rhomboidal five-body problem (3), which are simultaneously $S_{i}$-symmetric for $i=1,2,3$.

Proof: Assume that $\varphi(s)$ is a $S_{i}$-symmetric periodic solution of period $S$ for $i=1,2,3$. Then there exist times $s_{1}, s_{2}$, and $s_{3}$ with $s_{1}, s_{2}, s_{3} \in[0, S / 2)$ such that

$$
\xi_{1}\left(s_{1}\right)=\eta_{2}\left(s_{1}\right)=0, \quad \xi_{2}\left(s_{2}\right)=\eta_{1}\left(s_{2}\right)=0, \quad \eta_{1}\left(s_{3}\right)=\eta_{2}\left(s_{3}\right)=0 .
$$

We assume that $s_{1}=0$. This is not restrictive because system (3) is autonomous, and consequently the origin of time can be chosen arbitrarily. Then, since the orbit is in particular $S_{12}$-symmetric, from Proposition 2, $s_{2}=S / 4$. Similarly, since it is also $S_{13}$-symmetric, again from Proposition 2, $s_{3}=S / 4$. The fact that $s_{2}=s_{3}$ is a contradiction, so we have proved the result.

\section{SYMMETRIC PERIODIC SOLUTIONS FOR $\mu=0$}

For $\mu=0$ system (3) becomes

$$
\begin{array}{ll}
\frac{d \xi_{1}}{d s}=\frac{\eta_{1} \xi_{2}^{2}}{2 \nu_{1}}, & \frac{d \eta_{1}}{d s}=-\frac{\xi_{1} \eta_{2}^{2}}{2 \nu_{2}}+8 h \xi_{1} \xi_{2}^{2}+16 \nu_{2} \xi_{1}, \\
\frac{d \xi_{2}}{d s}=\frac{\eta_{2} \xi_{1}^{2}}{2 \nu_{2}}, & \frac{d \eta_{2}}{d s}=-\frac{\xi_{2} \eta_{1}^{2}}{2 \nu_{1}}+8 h \xi_{1}^{2} \xi_{2}+16 \nu_{1} \xi_{2},
\end{array}
$$

and the Hamiltonian $K$ goes over to

$$
K=\frac{\eta_{1}^{2} \xi_{2}^{2}}{4 \nu_{1}}+\frac{\eta_{2}^{2} \xi_{1}^{2}}{4 \nu_{2}}-8 \nu_{2} \xi_{1}^{2}-8 \nu_{1} \xi_{2}^{2}-4 h \xi_{1}^{2} \xi_{2}^{2}
$$

The Hamiltonian $H$ for $\mu=0$ can be written as

$$
H=H_{1}\left(x, p_{x}\right)+H_{2}\left(y, p_{y}\right)=\left(\frac{p_{x}^{2}}{4 \nu_{1}}-\frac{2 \nu_{1}}{x}\right)+\left(\frac{p_{y}^{2}}{4 \nu_{2}}-\frac{2 \nu_{2}}{y}\right) .
$$

We note that $H_{1}\left(x, p_{x}\right)$ and $H_{2}\left(y, p_{y}\right)$ are two fist integrals of the nonregularized problem, so they are constant along the solutions in the intervals between two consecutive zeros of $x$ and $y$.

The flow of the rhomboidal five-body problem on the energy level $H=h$ for some constant $h$ is obtained from the flow of the Hamiltonian $H_{1}\left(x, p_{x}\right)$ on the energy level $H_{1}=h_{1}$ and the flow of the Hamiltonian $H_{2}\left(y, p_{y}\right)$ on the energy level $H_{2}=h_{2}$ with $h=h_{1}+h_{2}$.

The Hamiltonian $H_{1}\left(x, p_{x}\right)$ in the Levi-Civita coordinates $\left(\xi_{1}, \eta_{1}\right)$ is given by 


$$
H_{1}=\frac{\eta_{1}^{2}}{16 \nu_{1} \xi_{1}^{2}}-\frac{2 \nu_{1}}{\xi_{1}^{2}}=h_{1},
$$

and the Hamiltonian $H_{2}\left(y, p_{y}\right)$ in the Levi-Civita coordinates $\left(\xi_{2}, \eta_{2}\right)$ is

$$
H_{2}=\frac{\eta_{2}^{2}}{16 \nu_{2} \dot{\xi}_{2}^{2}}-\frac{2 \nu_{2}}{\xi_{2}^{2}}=h_{2}
$$

Let $\left(\xi_{1}, \xi_{2}, \eta_{1}, \eta_{2}\right)$ be a solution of system (4) satisfying the energy relation $K=0$ (i.e., $H=h$ ), we define a new time variable $\sigma$ as follows:

$$
\frac{d \sigma}{d s}=\xi_{2}^{2}, \quad \text { or equivalently } \quad \frac{d t}{d \sigma}=4 \xi_{1}^{2} .
$$

The Hamiltonian $K$ in the new time variable $\sigma$ can be written as

$$
K_{1}=\frac{1}{\xi_{2}^{2}} K=\frac{\eta_{1}^{2}}{4 \nu_{1}}-8 \nu_{1}-4 h_{1} \xi_{1}^{2}+\left(\frac{\eta_{2}^{2}}{4 \nu_{2} \xi_{2}^{2}}-\frac{8 \nu_{2}}{\xi_{2}^{2}}-4 h_{2}\right) \xi_{1}^{2}=\frac{\eta_{1}^{2}}{4 \nu_{1}}-8 \nu_{1}-4 h_{1} \xi_{1}^{2} .
$$

Then $\xi_{1}, \eta_{1}$ satisfy the system of differential equations associated to the Hamiltonian $K_{1}$

$$
\frac{d \xi_{1}}{d \sigma}=\frac{\eta_{1}}{2 \nu_{1}}, \quad \frac{d \eta_{1}}{d \sigma}=8 h_{1} \xi_{1}
$$

We are only interested in the periodic solutions of system (6). Thus we must consider only negative values of $h_{1}$. Then, fixed $h_{1}<0$, system (6) can be integrated directly and the solution $\left(\xi_{1}(\sigma), \eta_{1}(\sigma)\right)$ of system (6) with initial conditions

$$
\xi_{1}(0)=\xi_{10}^{*}, \quad \eta_{1}(0)=\eta_{10}^{*},
$$

is

$$
\xi_{1}(\sigma)=\xi_{10}^{*} \cos \left(w_{1} \sigma\right)+\frac{\eta_{10}^{*}}{2 w_{1} \nu_{1}} \sin \left(w_{1} \sigma\right), \quad \eta_{1}(\sigma)=\eta_{10}^{*} \cos \left(w_{1} \sigma\right)-2 w_{1} \nu_{1} \xi_{10}^{*} \sin \left(w_{1} \sigma\right),
$$

where $w_{1}=2 \sqrt{-h_{1} / \nu_{1}}$.

We note that the solution (8) is a periodic solution of system (6) with period $\bar{\sigma}=2 \pi / w_{1}$. Since we are interested in the periodic solution (8) satisfying the energy relation $K_{1}=0$, by Eq. (5), its period in the real time $t$ is given by

$$
T_{1}\left(h_{1}, \nu_{1}\right)=\int_{0}^{\bar{\sigma}} 4 \xi_{1}^{2}(\sigma) d \sigma=4 \pi\left(-\frac{\nu_{1}}{h_{1}}\right)^{3 / 2} .
$$

Now we introduce a new time $\tau$ with

$$
\frac{d \tau}{d s}=\xi_{1}^{2}, \quad \text { or equivalently } \quad \frac{d t}{d \tau}=4 \xi_{2}^{2} .
$$

Then $\xi_{2}, \eta_{2}$ are functions of the new time $\tau$ via the Hamiltonian system

$$
\frac{d \xi_{2}}{d \tau}=\frac{\eta_{2}}{2 \nu_{2}}, \quad \frac{d \eta_{2}}{d \tau}=8 h_{2} \xi_{2}
$$

with Hamiltonian 
TABLE I. Period of $\varphi(s)$.

\begin{tabular}{lccc}
\hline \hline \multicolumn{1}{c}{ Time $t$} & Time $\sigma$ & Time $\tau$ & Time $s$ \\
\hline$T=p T_{1}\left(h_{1}, \nu_{1}\right)=q T_{2}\left(h_{2}, \nu_{2}\right)$ & $\sigma^{*}=p \bar{\sigma}$ & $\tau^{*}=q \bar{\tau}$ & $S^{*}=s(T)$ \\
$T / 4$ & $\sigma^{*} / 4$ & $\tau^{*} / 4$ & $S^{*} / 4$ \\
\hline \hline
\end{tabular}

$$
K_{2}=\frac{1}{\xi_{1}^{2}} K=\frac{\eta_{2}^{2}}{4 \nu_{2}}-8 \nu_{2}-4 h_{2} \xi_{2}^{2}
$$

Moreover, fixed $h_{2}<0$, the solution $\left(\xi_{2}(\tau), \eta_{1}(\tau)\right)$ of system (10) with initial conditions

$$
\xi_{2}(0)=\xi_{20}^{*}, \quad \eta_{2}(0)=\eta_{20}^{*},
$$

is given by

$$
\xi_{2}(\tau)=\xi_{20}^{*} \cos \left(w_{2} \tau\right)+\frac{\eta_{20}^{*}}{2 w_{2} \nu_{2}} \sin \left(w_{2} \tau\right), \quad \eta_{2}(\tau)=\eta_{20}^{*} \cos \left(w_{2} \tau\right)-2 w_{2} \nu_{2} \xi_{20}^{*} \sin \left(w_{2} \tau\right),
$$

where $w_{2}=2 \sqrt{-h_{2} / \nu_{2}}$.

The solution (12) is periodic of period $\bar{\tau}=2 \pi / w_{2}$. Moreover, if the solution (12) satisfies the energy relation $K_{2}=0$, then, by Eq. (9), the period of the solution (12) in the real time $t$ is given by

$$
T_{2}\left(h_{2}, \nu_{2}\right)=\int_{0}^{\bar{\tau}} 4 \xi_{2}^{2}(\tau) d \tau=4 \pi\left(-\frac{\nu_{2}}{h_{2}}\right)^{3 / 2} .
$$

Proposition 4: Let $\left(\xi_{1}(\sigma), \eta_{1}(\sigma)\right)$ be a periodic solution of system (6), for a fixed $h_{1}<0$, with initial conditions (7) and period $\bar{\sigma}=2 \pi / w_{1}$ that satisfies $K_{1}=0$. Let $\left(\xi_{2}(\tau), \eta_{2}(\tau)\right)$ be the periodic solution of system (10), for a fixed $h_{2}<0$, with initial conditions (11) and period $\bar{\tau}=2 \pi / w_{2}$ that satisfies $K_{2}=0$. Assume that $h=h_{1}+h_{2}$, and that $\sigma(s)$ and $\tau(s)$ are given by Eqs. (5) and (9), respectively, where we choose $\sigma(0)=\tau(0)=0$. Suppose that there is no $s \in \mathbb{R}$ such that $\xi_{1}(\sigma(s))$ $=\xi_{2}(\tau(s))=0$. Then the following statements hold:

(a) $\varphi(s)=\left(\xi_{1}(\sigma(s)), \xi_{2}(\tau(s)), \eta_{1}(\sigma(s)), \eta_{2}(\tau(s))\right.$ is a solution of system (4) with initial conditions $\xi_{1}(0)=\xi_{10}^{*}, \xi_{2}(0)=\xi_{20}^{*}, \eta_{1}(0)=\eta_{10}^{*}$, and $\eta_{2}(0)=\eta_{20}^{*}$ that satisfies $K=0$.

(b) If $h_{1}=(p / q)^{2 / 3} \nu_{1} h_{2} / \nu_{2}$ for some $p, q \in \mathbb{N}$ coprime, then $\varphi(s)$ is a periodic solution of system (4).

(c) Assume that $s(t)$ is given by the inverse function of $t=\int_{0}^{s} 4 \xi_{1}^{2}(\rho) \xi_{2}^{2}(\rho) d \rho$. Under the hypotheses of statement $(b)$, the period and the quarter of the period of the periodic solution $\varphi(s)$ using the different times $t, \sigma, \tau$, and $s$ is given in Table I.

Proof: Statement (a) follows easily from the definitions of $\left(\xi_{1}(\sigma), \eta_{1}(\sigma)\right)$ and $\left(\xi_{2}(\tau), \eta_{2}(\tau)\right)$ together with the definitions of $\sigma(s)$ and $\tau(s)$.

We have seen that, in the time $t,\left(\xi_{1}(\sigma), \eta_{1}(\sigma)\right)$ and $\left(\xi_{2}(\tau), \eta_{2}(\tau)\right)$ are periodic solutions of periods $T_{1}\left(h_{1}, \nu_{1}\right)$ and $T_{2}\left(h_{2}, \nu_{2}\right)$, respectively. Thus, in order to have a periodic solution of system (4) we need that

$$
p T_{1}\left(h_{1}, \nu_{1}\right)=q T_{2}\left(h_{2}, \nu_{2}\right),
$$

for some $p, q \in \mathbb{N}$ coprime. Solving Eq. (13) with respect to $h_{1}$, we get that $h_{1}=(p / q)^{2 / 3} \nu_{1} h_{2} / \nu_{2}$. So, statement (b) is proved.

Now we see that the time $t=T / 4$ corresponds to the time $\sigma=\sigma^{*} / 4$. In a similar way we can see that the time $t=T / 4$ corresponds to the time $\tau=\tau^{*} / 4$ and $s=S^{*} / 4$. 
We note that system (6) is invariant under the symmetry $\left(\xi_{1}, \eta_{1}, \sigma\right) \rightarrow\left(-\xi_{1}, \eta_{1},-\sigma\right)$. This means that $\xi_{1}(\sigma)=-\xi_{1}(-\sigma)$. So $\xi_{1}^{2}(\sigma)$ is an even function. On the other hand, it is easy to see that $\xi_{1}^{2}(\sigma)$ is a periodic function of period $\bar{\sigma} / 2$. Then, from Eq. (5), we have that

$$
T_{1}=\int_{0}^{\bar{\sigma}} 4 \xi_{1}^{2}(\sigma) d \sigma=2 \int_{0}^{\bar{\sigma} / 2} 4 \xi_{1}^{2}(\sigma) d \sigma=4 \int_{0}^{\bar{\sigma} / 4} 4 \xi_{1}^{2}(\sigma) d \sigma
$$

Moreover, it is clear that

$$
\int_{0}^{\bar{\sigma} / 4} 4 \xi_{1}^{2}(\sigma) d \sigma=\int_{\bar{\sigma} / 4}^{\bar{\sigma} / 2} 4 \xi_{1}^{2}(\sigma) d \sigma=\frac{T_{1}}{4}
$$

Consequently

$$
t\left(\sigma^{*} / 4\right)=\int_{0}^{p \bar{\sigma} / 4} 4 \xi_{1}^{2}(\sigma) d \sigma=p \int_{0}^{\bar{\sigma} / 4} 4 \xi_{1}^{2}(\sigma) d \sigma=p \frac{T_{1}}{4}=\frac{T}{4} .
$$

Therefore, the time $t=T / 4$ corresponds to $\sigma=\sigma^{*} / 4$. In short, statement (c) is proved.

We remark that the number $p$ in Proposition 4 represents the number of triple collisions between $m_{0}, m_{1}$, and $m_{2}$ during a period, whereas $q$ represents the number of triple collisions between $m_{0}, m_{3}$, and $m_{4}$.

We are interested in symmetric periodic solutions of system (4) satisfying the energy relation $K=0$ with $h=h_{1}+h_{2}$. In the next proposition we give initial conditions for those symmetric periodic solutions.

Proposition 5: The following statements hold:

(a) If $p$ and $q$ are odd, then the solution $\varphi(s)$ given by Proposition 4 with initial conditions

$$
\begin{gathered}
\text { either } \xi_{10}^{*}=0, \xi_{20}^{*}=\sqrt{-2 \nu_{2} / h_{2}}, \eta_{10}^{*}=4 \sqrt{2} \nu_{1}, \eta_{20}^{*}=0 ; \\
\text { or } \xi_{10}^{*}=\sqrt{-2 \nu_{1} / h_{1}}, \xi_{20}^{*}=0, \eta_{10}^{*}=0, \eta_{20}^{*}=4 \sqrt{2} \nu_{2} ;
\end{gathered}
$$

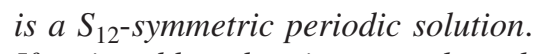

(b) If $p$ is odd and $q$ is even, then the solution $\varphi(s)$ given by Proposition 4 with initial conditions

$$
\begin{aligned}
& \text { either } \xi_{10}^{*}=0, \xi_{20}^{*}=\sqrt{-2 \nu_{2} / h_{2}}, \eta_{10}^{*}=4 \sqrt{2} \nu_{1}, \eta_{20}^{*}=0 ; \\
& \text { or } \xi_{10}^{*}=\sqrt{-2 \nu_{1} / h_{1}}, \xi_{20}^{*}=\sqrt{-2 \nu_{2} / h_{2}}, \eta_{10}^{*}=0, \eta_{20}^{*}=0 ;
\end{aligned}
$$

is a $S_{13}$-symmetric periodic solution.

(c) If $p$ is even and $q$ is odd, then the solution $\varphi(s)$ given by Proposition 4 with initial conditions

$$
\begin{aligned}
& \text { either } \xi_{10}^{*}=\sqrt{-2 \nu_{1} / h_{1}}, \xi_{20}^{*}=0, \eta_{10}^{*}=0, \eta_{20}^{*}=4 \sqrt{2} \nu_{2} ; \\
& \text { or } \xi_{10}^{*}=\sqrt{-2 \nu_{1} / h_{1}}, \xi_{20}^{*}=\sqrt{-2 \nu_{2} / h_{2}}, \eta_{10}^{*}=0, \eta_{20}^{*}=0 ;
\end{aligned}
$$

is a $S_{23}$-symmetric periodic solution.

Proof: Solving $K_{1}=0$ and $K_{2}=0$ for the initial conditions of the $S_{12}$-symmetric periodic orbits given in Proposition 2(a) we get the initial conditions of statement (a). In these initial conditions we have only considered the positive determination in the squareroots due to the fact that the Levi-Civita transformation duplicates the orbits. The proof follows from the evaluation of the solution $\varphi(s)=\left(\xi_{1}(\sigma(s)), \xi_{2}(\tau(s)), \eta_{1}(\sigma(s)), \eta_{2}(\tau(s))\right.$ with these initial conditions at times $s=0$ and 
$s=S^{*} / 4$. We note that by Table I $\varphi\left(S^{*} / 4\right)=\left(\xi_{1}(p \bar{\sigma} / 4), \xi_{2}(q \bar{\tau} / 4), \eta_{1}(p \bar{\sigma} / 4), \eta_{2}(q \bar{\tau} / 4)\right)$. This completes the proof of statement (a). The other statements follow similarly.

\section{CONTINUATION OF SYMMETRIC PERIODIC SOLUTIONS}

In this section using the continuation method of Poincare (see, for instance, Ref. 10) we shall continue the symmetric periodic orbits of the rhomboidal five-body problem (3) from $\mu=0$ to symmetric periodic orbits of system (3) for $\mu>0$ sufficiently small.

\section{A. The $S_{12}$-symmetric periodic solutions}

We denote by $\varphi\left(s ; 0, \xi_{20}, \eta_{10}, 0, \mu\right)=\left(\xi_{1}\left(s ; \xi_{20}, \eta_{10}, \mu\right), \xi_{2}\left(s ; \xi_{20}, \eta_{10}, \mu\right), \eta_{1}\left(s ; \xi_{20}, \eta_{10}, \mu\right)\right.$, $\left.\eta_{2}\left(s ; \xi_{20}, \eta_{10}, \mu\right)\right)$ the solution of (3), for fixed values of $\nu_{1}>0, \nu_{2}>0$, and $h<0$, with initial conditions $\quad \xi_{1}(0)=0, \quad \xi_{2}(0)=\xi_{20}, \quad \eta_{1}(0)=\eta_{10}, \quad$ and $\quad \eta_{2}(0)=0$. From Proposition 2(a), $\varphi\left(s ; 0, \xi_{20}, \eta_{10}, 0, \mu\right)$ is a $S_{12}$-symmetric periodic solution of the rhomboidal five-body problem with period $S$ satisfying the energy relation $K=0$ if and only if

$$
\xi_{2}\left(S / 4 ; \xi_{20}, \eta_{10}, \mu\right)=0, \quad \eta_{1}\left(S / 4 ; \xi_{20}, \eta_{10}, \mu\right)=0, \quad K\left(\xi_{20}, \eta_{10}, \mu\right)=0 .
$$

We solve equation $K\left(\xi_{20}, \eta_{10}, \mu\right)=0$ with respect the variable $\eta_{10}$ obtaining in this way

$$
\eta_{10}=2 \sqrt{2} \nu_{1} \sqrt{4+\mu \nu_{1}} .
$$

So $\varphi\left(s ; 0, \xi_{20}, \eta_{10}, 0, \mu\right)$ is a $S_{12}$-symmetric periodic solution of the rhomboidal five-body problem with period $S$ satisfying the energy relation $K=0$ if and only if

$$
\xi_{2}\left(S / 4 ; \xi_{20}, \mu\right)=0, \quad \eta_{1}\left(S / 4 ; \xi_{20}, \mu\right)=0 .
$$

Notice that we have omitted the dependence with respect to $\eta_{10}$, which is given by Eq. (14).

Assume that $p=2 \ell+1, q=2 k+1$ for some $\ell, k \in \mathbb{N} \cup\{0\}$ and that $h_{1}^{*}$ and $h_{2}^{*}$ verify that $h$ $=h_{1}^{*}+h_{2}^{*}$ and $h_{1}^{*}=(p / q)^{2 / 3} \nu_{1} h_{2}^{*} / \nu_{2}$. By Propositions 4 and $5(\mathrm{a})$, we see that $S=S^{*}=s\left(p T_{1}\left(h_{1}^{*}\right)\right)$ $=s\left(q T_{2}\left(h_{2}^{*}\right)\right), \xi_{20}=\xi_{20}^{*}=\sqrt{-2 \nu_{2} / h_{2}^{*}}$, is a solution of system (15) for $\mu=0$. This solution correspond to the known $S_{12}$-symmetric periodic solution $\varphi\left(s ; 0, \xi_{20}^{*}, \eta_{10}^{*}, 0,0\right)$ of system (3), for $\mu=0$ where $\eta_{10}^{*}=4 \sqrt{2} \nu_{1}$. Our aim is to continue this solution of system (15) for $\mu=0$ to $\mu>0$ sufficiently small.

Applying the implicit function theorem to system (15) in a neighborhood of the known solution we have that if

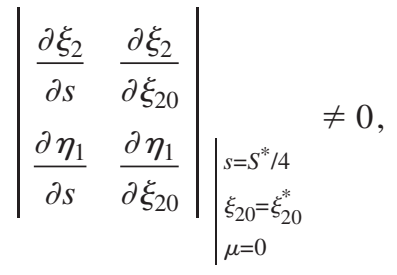

then we can find unique analytic functions $\xi_{20}=\xi_{20}(\mu), S=S(\mu)$ defined for $\mu \geqslant 0$ sufficiently small, such that

(i) $\xi_{20}(0)=\xi_{20}^{*}, S(0)=S^{*}$,

(ii) $\varphi\left(s ; 0, \xi_{20}, \eta_{10}, 0, \mu\right)$ with $\xi_{20}=\xi_{20}(\mu)$ and $\eta_{10}$ given by (14) is a $S_{12}$-symmetric periodic solution of system (3) with period $S=S(\mu)$ that satisfies the energy relation $K=0$.

The derivatives $\partial \xi_{2} / \partial s$ and $\partial \eta_{1} / \partial s$ evaluated at $s=S^{*} / 4, \xi_{20}=\xi_{20}^{*}$ and $\mu=0$ can be obtained directly from system (3) for $\mu=0$ [i.e., system (4)], evaluating the right hand of the system at the solution $\varphi\left(s ; 0, \xi_{20}^{*}, \eta_{10}^{*}, 0,0\right)$ at time $s=S^{*} / 4$. Then 


$$
\left.\frac{\partial \xi_{2}}{\partial s}\right|_{\substack{\xi_{20}=\xi_{20}^{*} \\ \mu=0}} ^{s=S^{*} / 4}=\left.\frac{1}{2 \nu_{2}} \eta_{2} \xi_{1}^{2}\right|_{\substack{s=S^{*} / 4 \\ \xi_{20}=\xi_{20}^{*} \\ \mu=0}}=\frac{4(-1)^{k} \sqrt{2} \nu_{2}}{h_{2}^{*}}\left(\frac{q}{p}\right)^{2 / 3} \neq 0
$$

and

$$
\left.\frac{\partial \eta_{1}}{\partial s}\right|_{\substack{s=S^{*} / 4 \\ \xi_{20}=\xi_{20}^{*} \\ \mu=0}}=\left.8 h_{1}^{*} \xi_{1} \xi_{2}^{2}\right|_{s=S^{*} / 4}=0
$$

It only remains to compute the value of $\partial \eta_{1} / \partial \xi_{20}$ evaluated at $s=S^{*} / 4, \xi_{20}=\xi_{20}^{*}, \mu=0$. This value is given by the derivative, evaluated at $s=S^{*} / 4$ and $\xi_{20}=\xi_{20}^{*}$, of the solution $\eta_{1}\left(\sigma(s) ; 0, \xi_{20}, \eta_{10}^{*}, 0,0\right)$ with respect to $\xi_{20}$, where $\eta_{1}\left(\sigma(s) ; 0, \xi_{20}, \eta_{10}^{*}, 0,0\right)$ is the solution of system (4) with initial conditions $\xi_{1}(0)=0, \xi_{2}(0)=\xi_{20}, \eta_{1}(0)=\eta_{10}^{*}$, and $\eta_{2}(0)=0$ satisfying the energy relation $K=0$ [see Proposition 4(a)]. Then

$$
\left.\frac{\partial \eta_{1}\left(\sigma(s) ; 0, \xi_{20}, \eta_{10}^{*}, 0,0\right)}{\partial \xi_{20}}\right|_{\substack{s=S^{*} / 4 \\ \xi_{20}=\xi_{20}^{*}}}=\left.\left(\frac{\partial \eta_{1}}{\partial \sigma} \frac{\partial \sigma(s)}{\partial \xi_{20}}+\frac{\partial \eta_{1}}{\partial \xi_{20}}\right)\right|_{\substack{s=S^{*} / 4 \\ \xi_{20}=\xi_{20}^{*}}}
$$

From Eqs. (5) and (9), we have that the times $\sigma$ and $\tau$ are related by

$$
\xi_{1}^{2}(\sigma) d \sigma=\xi_{2}^{2}(\tau) d \tau
$$

Integrating this equation over the solutions (8) and (12) with the corresponding initial conditions and assuming that when $\sigma(0)=\tau(0)=0$, we have that $\sigma(s)$ and $\tau(s)$ are related by the equation

$$
\frac{4 \sigma(s)}{w_{1}^{2}}-\frac{\xi_{20}^{2} \tau(s)}{2}-\frac{2 \sin \left(2 w_{1} \sigma(s)\right)}{w_{1}^{3}}-\frac{\xi_{20}^{2} \sin \left(2 w_{2} \tau(s)\right)}{4 w_{2}}=0 .
$$

Since $\varphi\left(s ; 0, \xi_{20}, \eta_{10}^{*}, 0,0\right)$ must be a solution of system (4), by Proposition 4 we have that $K_{1}$ $=0$ and $K_{2}=0$, so

$$
w_{2}=2 \sqrt{-h_{2} / \nu_{2}}, \quad \text { with } \quad h_{2}=-2 \nu_{2} / \xi_{20}^{2}
$$

and

$$
w_{1}=2 \sqrt{-h_{1} / \nu_{1}}, \quad \text { with } \quad h_{1}=h-h_{2} .
$$

Then derivating implicitly Eq. (17) with respect to $\xi_{20}$ we obtain

$$
\left.\frac{\partial \sigma(s)}{\partial \xi_{20}}\right|_{\substack{s=S^{*} / 4 \\ \xi_{20}=\xi_{20}^{*}}}=\frac{\pi q}{8 \sqrt{2} \nu_{1}}\left(3\left(\frac{p}{q}\right)^{2 / 3} \nu_{1}+\nu_{2}\right) .
$$

On the other hand, from systems (6) and (8) we have that

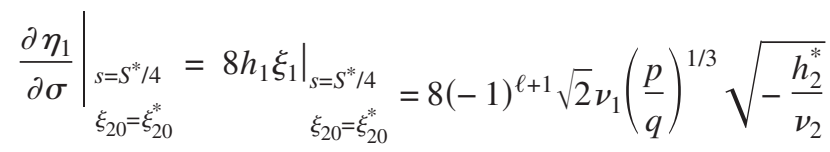

and

$$
\left.\frac{\partial \eta_{1}}{\partial \xi_{20}}\right|_{\substack{s=S^{*} / 4 \\ \xi_{20}=\xi_{20}^{*}}}=2(-1)^{\ell} p h_{2}^{*} \pi\left(\frac{q}{p}\right)^{2 / 3} \sqrt{-\frac{\nu_{2}}{h_{2}^{*}}}
$$

Hence 


$$
\left.\frac{\partial \eta_{1}\left(\sigma(s) ; 0, \xi_{20}, \eta_{10}^{*}, 0,0\right)}{\partial \xi_{20}}\right|_{\substack{s=S^{*} / 4 \\ \xi_{20}=\xi_{20}^{*}}}=3(-1)^{\ell+1} \pi \sqrt{-\frac{h_{2}^{*}}{\nu_{2}}}\left(p \nu_{1}+\left(\frac{p}{q}\right)^{1 / 3} q \nu_{2}\right) .
$$

So, this derivative is different from zero. Hence, the determinant (16) is not zero. In short, we have proved the following result.

Theorem 6: Given $\nu_{1}>0, \nu_{2}>0, h<0$ and $p$ and $q$ odd positive integers, the $S_{12}$-symmetric periodic solution of the rhomboidal five-body problem (3) for $\mu=0$ with initial conditions $\xi_{1}(0)$ $=0, \xi_{2}(0)=\sqrt{-2 \nu_{2} / h_{2}^{*}}, \eta_{1}(0)=4 \sqrt{2} \nu_{1}$, and $\eta_{2}(0)=0$ where $h_{2}^{*}=h \nu_{2} /\left((p / q)^{2 / 3} \nu_{1}+\nu_{2}\right)$, can be continued to a $\mu$-parameter family of $S_{12}$-symmetric periodic orbits of the rhomboidal five-body problem (3)for $\mu>0$ sufficiently small.

\section{B. The $S_{23}$-symmetric periodic solutions}

We denote by $\varphi\left(s ; \xi_{10}, \xi_{20}, 0,0, \mu\right)=\left(\xi_{1}\left(s ; \xi_{10}, \xi_{20}, \mu\right), \xi_{2}\left(s ; \xi_{10}, \xi_{20}, \mu\right), \eta_{1}\left(s ; \xi_{10}, \xi_{20}, \mu\right)\right.$, $\left.\eta_{2}\left(s ; \xi_{10}, \xi_{20}, \mu\right)\right)$ the solution of system (3), for fixed values of $\nu_{1}>0, \nu_{2}>0$, and $h<0$, with initial conditions $\xi_{1}(0)=\xi_{10}, \quad \xi_{2}(0)=\xi_{20}, \quad \eta_{1}(0)=0$, and $\eta_{2}(0)=0$. From Proposition 2(c), $\varphi\left(s ; \xi_{10}, \xi_{20}, 0,0, \mu\right)$ is a $S_{23}$-symmetric periodic solution of the rhomboidal five-body problem with period $S$ satisfying the energy relation $K=0$ if and only if

$$
\xi_{2}\left(S / 4 ; \xi_{10}, \xi_{20}, \mu\right)=0, \quad \eta_{1}\left(S / 4 ; \xi_{10}, \xi_{20}, \mu\right)=0, \quad K\left(\xi_{10}, \xi_{20}, \mu\right)=0 .
$$

We solve equation $K\left(\xi_{10}, \xi_{20}, \mu\right)=0$ with respect to the variable $\xi_{20}$ obtaining in this way $\xi_{20}=\xi_{20}\left(\xi_{10}, \mu\right)$. In particular, $\xi_{20}\left(\xi_{10}, 0\right)=\sqrt{2 \nu_{2}} \xi_{10} / \sqrt{-2 \nu_{1}-h \xi_{10}^{2}}$. So $\varphi\left(s ; \xi_{10}, \xi_{20}, 0,0, \mu\right)$ is a $S_{23}$-symmetric periodic solution of the rhomboidal five-body problem with period $S$ satisfying the energy relation $K=0$ if and only if

$$
\xi_{2}\left(S / 4 ; \xi_{10}, \mu\right)=0, \quad \eta_{1}\left(S / 4 ; \xi_{10}, \mu\right)=0 .
$$

Assume that $p=2 \ell, q=2 k+1$ for some $\ell \in \mathbb{N}$ and $k \in \mathbb{N} \cup\{0\}$ and that $h_{1}^{*}$ and $h_{2}^{*}$ verify that $h=h_{1}^{*}+h_{2}^{*}$ and $h_{1}^{*}=(p / q)^{2 / 3} \nu_{1} h_{2}^{*} / \nu_{2}$. By Propositions 4 and 5(c), we see that $S=S^{*}=s\left(p T_{1}\left(h_{1}^{*}\right)\right)$ $=s\left(q T_{2}\left(h_{2}^{*}\right)\right), \xi_{10}=\xi_{10}^{*}=\sqrt{-2 \nu_{1} / h_{1}^{*}}$ is a solution of system (18) for $\mu=0$. This solution corresponds to the known $S_{23}$-symmetric periodic solution $\varphi\left(s ; \xi_{10}^{*}, \xi_{20}^{*}, 0,0,0\right)$ of system (3), for $\mu=0$ where $\xi_{20}=\xi_{20}^{*}=\sqrt{-2 \nu_{2} / h_{2}^{*}}$. Our aim is to continue this solution of system (18) for $\mu=0$ to $\mu>0$ sufficiently small.

Applying the implicit function theorem to system (18) in a neighborhood of the known solution we have that if

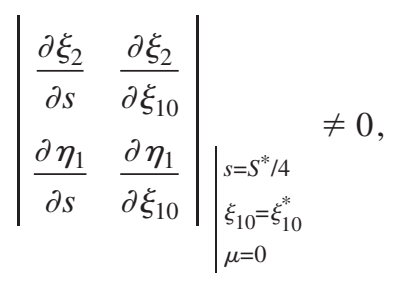

then we can find unique analytic functions $\xi_{10}=\xi_{10}(\mu), S=S(\mu)$ defined for $\mu \geqslant 0$ sufficiently small, such that

(i) $\xi_{10}(0)=\xi_{10}^{*}, S(0)=S^{*}$,

(ii) $\varphi\left(s ; \xi_{10}, \xi_{20}, 0,0, \mu\right)$ with $\xi_{10}=\xi_{10}(\mu)$ and $\xi_{20}=\xi_{20}\left(\xi_{10}(\mu), \mu\right)$ is a $S_{23}$-symmetric periodic solution of system (3) with period $S=S(\mu)$ that satisfies the energy relation $K=0$.

Working as for the $S_{12}$-symmetry we get that 


$$
\left.\frac{\partial \xi_{2}}{\partial s}\right|_{\substack{s=S^{*} / 4 \\ \xi_{10}=\xi_{10}^{*}}} \neq 0, \text { and }\left.\quad \frac{\partial \eta_{1}}{\partial s}\right|_{\substack{s=S^{*} / 4 \\ \xi_{10}=\xi_{10}^{*} \\ \mu=0}}=0
$$

It only remains to compute the value of $\partial \eta_{1} / \partial \xi_{10}$ evaluated at $s=S^{*} / 4, \xi_{10}=\xi_{10}^{*}, \mu=0$. This value is given by the derivative, evaluated at $s=S^{*} / 4$ and $\xi_{10}=\xi_{10}^{*}$, of the solution $\eta_{1}\left(\sigma(s) ; \xi_{10}, \xi_{20}, 0,0,0\right)$ with respect to $\xi_{10}$, where $\eta_{1}\left(\sigma(s) ; \xi_{10}, \xi_{20}, 0,0,0\right)$ is the solution of system (4) with initial conditions $\xi_{1}(0)=\xi_{10}, \xi_{2}(0)=\xi_{20}\left(\xi_{10}, 0\right), \eta_{1}(0)=0$, and $\eta_{2}(0)=0$ satisfying the energy relation $K=0$ [see Proposition 4(c)]. Then

$$
\begin{aligned}
\left.\frac{\partial \eta_{1}\left(\sigma(s) ; 0, \xi_{10}, \xi_{20}, 0,0,0\right)}{\partial \xi_{10}}\right|_{\substack{s=S^{*} / 4 \\
\xi_{10}=\xi_{10}^{*}}}=\left.\left(\frac{\partial \eta_{1}}{\partial \sigma} \frac{\partial \sigma(s)}{\partial \xi_{10}}+\frac{\partial \eta_{1}}{\partial \xi_{10}}\right)\right|_{\substack{s=S^{*} / 4 \\
\xi_{10}=\xi_{10}^{*}}} \\
=\frac{3(-1)^{\ell+1} h_{2}^{*} p \pi \nu_{1}}{q \nu_{2}^{2}}\left(p \nu_{1}+\left(\frac{p}{q}\right)^{1 / 3} q \nu_{2}\right),
\end{aligned}
$$

so this derivative is different from zero. Hence, the determinant (19) is not zero. In short, we have proved the following result.

Theorem 7: Given $\nu_{1}>0, \nu_{2}>0 h<0, p$ even and $q$ odd positive integers, the $S_{23}$-symmetric periodic solution of the rhomboidal five-body problem (3) for $\mu=0$ with initial conditions $\xi_{1}(0)$ $=\sqrt{-2 \nu_{1} / h_{1}^{*}}, \quad \xi_{2}(0)=\sqrt{-2 \nu_{2} / h_{2}^{*}}, \quad \eta_{1}(0)=0, \quad$ and $\eta_{2}(0)=0$, where $h_{2}^{*}=h \nu_{2} /\left((p / q)^{2 / 3} \nu_{1}+\nu_{2}\right), \quad h_{1}^{*}$ $=h(p / q)^{2 / 3} \nu_{1} /\left((p / q)^{2 / 3} \nu_{1}+\nu_{2}\right)$, can be continued to a $\mu$-parameter family of $S_{23}$-symmetric periodic orbits of the rhomboidal five-body problem (3) for $\mu>0$ sufficiently small.

\section{The $S_{13}$-symmetric periodic solutions}

Let $\varphi\left(s ; \xi_{10}, \xi_{20}, 0,0, \mu\right)$ be the solution of system (3), for fixed values of $\nu_{1}>0, \nu_{2}>0$, and $h<0$, defined as in Sec. V B. From Proposition 2(b), $\varphi\left(s ; \xi_{10}, \xi_{20}, 0,0, \mu\right)$ is a $S_{13}$-symmetric periodic solution of the rhomboidal five-body problem with period $S$ satisfying the energy relation $K=0$ if and only if

$$
\xi_{1}\left(S / 4 ; \xi_{10}, \xi_{20}, \mu\right)=0, \quad \eta_{2}\left(S / 4 ; \xi_{10}, \xi_{20}, \mu\right)=0, \quad K\left(\xi_{10}, \xi_{20}, \mu\right)=0 .
$$

Let $\xi_{20}=\xi_{20}\left(\xi_{10}, \mu\right)$ be the function defined in Sec. V B. Then $\varphi\left(s ; \xi_{10}, \xi_{20}, 0,0, \mu\right)$ is a $S_{13}$-symmetric periodic solution of the rhomboidal five-body problem with period $S$ satisfying the energy relation $K=0$ if and only if

$$
\xi_{1}\left(S / 4 ; \xi_{10}, \mu\right)=0, \quad \eta_{2}\left(S / 4 ; \xi_{10}, \mu\right)=0 .
$$

Assume that $p=2 \ell+1, q=2 k$ for some $\ell \in \mathbb{N} \cup\{0\}$ and $k \in \mathbb{N}$ and that $h_{1}^{*}$ and $h_{2}^{*}$ verify that $h=h_{1}^{*}+h_{2}^{*}$ and $h_{1}^{*}=(p / q)^{2 / 3} \nu_{1} h_{2}^{*} / \nu_{2}$. By Propositions 4 and $5(\mathrm{~b})$, we see that $S=S^{*}=s\left(p T_{1}\left(h_{1}^{*}\right)\right)$ $=s\left(q T_{2}\left(h_{2}^{*}\right)\right), \xi_{10}=\xi_{10}^{*}=\sqrt{-2 \nu_{1} / h_{1}^{*}}$ is a solution of system (20) for $\mu=0$. This solution correspond to the known $S_{13}$-symmetric periodic solution $\varphi\left(s ; \xi_{10}^{*}, \xi_{20}^{*}, 0,0,0\right)$ of system (3), for $\mu=0$ where $\xi_{20}=\xi_{20}^{*}=\sqrt{-2 \nu_{2} / h_{2}^{*}}$. Our aim is to continue this solution of system (20) for $\mu=0$ to $\mu>0$ sufficiently small.

Applying the implicit function theorem to system (20) in a neighborhood of the known solution we have that if 


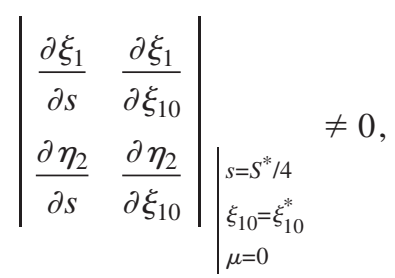

then we can find unique analytic functions $\xi_{10}=\xi_{10}(\mu), S=S(\mu)$ defined for $\mu \geqslant 0$ sufficiently small, such that

$$
\xi_{10}(0)=\xi_{10}^{*}, S(0)=S^{*}
$$

(ii) $\varphi\left(s ; \xi_{10}, \xi_{20}, 0,0, \mu\right)$ with $\xi_{10}=\xi_{10}(\mu)$ and $\xi_{20}=\xi_{20}\left(\xi_{10}(\mu), \mu\right)$ is a $S_{13}$-symmetric periodic solution of system (3) with period $S=S(\mu)$ that satisfies the energy relation $K=0$.

Working as for the $S_{12}$-symmetry we get that

$$
\left.\frac{\partial \xi_{1}}{\partial s}\right|_{\substack{s=S^{*} / 4 \\ \xi_{10}=\xi_{10}^{*} \\ \mu=0}} \neq 0, \quad \text { and }\left.\quad \frac{\partial \eta_{2}}{\partial s}\right|_{\substack{s=S^{*} / 4 \\ \xi_{10}=\xi_{10}^{*} \\ \mu=0}}=0 .
$$

It only remains to compute the value of $\partial \eta_{2} / \partial \xi_{10}$ evaluated at $s=S^{*} / 4, \xi_{10}=\xi_{10}^{*}, \mu=0$. This value is given by the derivative, evaluated at $s=S^{*} / 4$ and $\xi_{10}=\xi_{10}^{*}$, of the solution $\eta_{2}\left(\tau(s) ; \xi_{10}, \xi_{20}, 0,0,0\right)$ with respect to $\xi_{10}$, where $\eta_{2}\left(\tau(s) ; \xi_{10}, \xi_{20}, 0,0,0\right)$ is the solution of system (4) with initial conditions $\xi_{1}(0)=\xi_{10}, \xi_{2}(0)=\xi_{20}\left(\xi_{10}, 0\right), \eta_{1}(0)=0$, and $\eta_{2}(0)=0$ satisfying the energy relation $K=0$ [see Proposition 4(b)]. Then

$$
\begin{aligned}
\left.\frac{\partial \eta_{2}\left(\tau(s) ; \xi_{10}, \xi_{20}, 0,0,0\right)}{\partial \xi_{10}}\right|_{\substack{s=S^{*} / 4 \\
\xi_{10}=\xi_{10}^{*}}} & =\left(\frac{\partial \eta_{2}}{\partial \tau} \frac{\partial \tau(s)}{\partial \xi_{10}}+\frac{\partial \eta_{2}}{\partial \xi_{10}}\right)_{\substack{s=S^{*} / 4 \\
\xi_{10}=\xi_{10}^{*}}} \\
& =\frac{(-1)^{k} h_{2} \pi}{\nu_{2}}\left(p \nu_{1}+\left(\frac{p}{q}\right)^{1 / 3} q\left(2 \nu_{1}+3 \nu_{2}\right)\right),
\end{aligned}
$$

so this derivative is different from zero. Hence, the determinant (21) is not zero. In short, we have proved the following result.

Theorem 8: Given $\nu_{1}>0, \nu_{2}>0, h<0, p$ odd and $q$ even positive integers, the $S_{13}$-symmetric periodic solution of the rhomboidal five-body problem (3) for $\mu=0$ with initial conditions $\xi_{1}(0)$ $=\sqrt{-2 \nu_{1} / h_{1}^{*}}, \quad \xi_{2}(0)=\sqrt{-2 \nu_{2} / h_{2}^{*}}, \quad \eta_{1}(0)=0, \quad$ and $\eta_{2}(0)=0$ where $h_{2}^{*}=h \nu_{2} /\left((p / q)^{2 / 3} \nu_{1}+\nu_{2}\right), \quad h_{1}^{*}$ $=h(p / q)^{2 / 3} \nu_{1} /\left((p / q)^{2 / 3} \nu_{1}+\nu_{2}\right)$, can be continued to a $\mu$-parameter family of $S_{13}$-symmetric periodic orbits of the rhomboidal five-body problem (3) for $\mu>0$ sufficiently small.

\section{ACKNOWLEDGMENTS}

The first author is partially supported by Grant No. MCYT-Spain MTM2005-06098-C02-01. The second author is partially supported by Grant Nos. MCYT-Spain MTM2005-06098-C02-01 and CIRIT-Spain 2005SGR 00550.

${ }^{1}$ R. Abraham and J. E. Marsden (Foundations of Mechanics, Benjamin, New York, 1978).

${ }^{2}$ V. I. Arnold, Mathematical Methods of Classical Mechanics (Springer-Verlag, Berlin, 1978).

${ }^{3}$ K. R. Meyer and G. R. Hall, An Introduction to Hamiltonian Dynamical Systems (Springer-Verlag, New York, 1991).

${ }^{4}$ V. Szebehely, Theory of Orbits (Academic, New York, 1967).

${ }^{5}$ R. Broucke and D. E. Walker, Celest. Mech. 21, 73-81 (1980).

${ }^{6}$ P. C. Kammeyer, Celest. Mech. 30, 329-342 (1983).

${ }^{7}$ J. Casasayas and J. Llibre, Mem. Am. Math. Soc. 52, 1-115 (1984).

${ }^{8}$ M. Santoprete, J. Math. Phys. 43, 3207-3219 (2002).

${ }^{9}$ M. Corbera and J. Llibre, Developments in Mathematical and Experimental Physics, Vol. C: Hydrodynamics and Dynamical Systems, ed. by Macias et al. (Kluwer Academic, Dordrecht, New York, 2002), pp. 117-141.

${ }^{10}$ C. L. Siegel and J. K. Moser, Lectures on Celestial Mechanics (Springer-Verlag, Berlin, 1971). 